\title{
Title: Magnitude of Preoperative Cervical Lordotic Compensation and C2-T3 Angle are Correlated to Increased Risk of Post-operative Sagittal Spinal Pelvic Malalignment in Adult Thoracolumbar Deformity Patients at 2-year Follow-up
}

\author{
Authors: Peter G. Passias ${ }^{\mathrm{a}}$, Alexandra Soroceanu ${ }^{\mathrm{b}}$, Justin Scheer ${ }^{\mathrm{c}}$, Sun Yang ${ }^{\mathrm{a}}$, Anthony \\ Boniello $^{\mathrm{a}}$, Justin S. Smith ${ }^{\mathrm{d}}$, Themistocles Protopsaltis ${ }^{\mathrm{a}}$, Han Jo Kim ${ }^{\mathrm{e}}$, Frank Schwab ${ }^{\mathrm{a}}$, \\ Munish Gupta ${ }^{\mathrm{f}}$, Eric Klineberg ${ }^{\mathrm{f}}$, Gregory Mundis ${ }^{\mathrm{g}}$, Renaud Lafage ${ }^{\mathrm{a}}$, Robert Hart ${ }^{\mathrm{h}}$ \\ Christopher Shaffrey ${ }^{c}$, Virginie Lafage ${ }^{\mathrm{a}}$, Christopher Ames ${ }^{\mathrm{i}}$, ISSG $^{\mathrm{j}}$
}

\footnotetext{
Affiliations:

${ }^{a}$ Orthopaedic Surgery, NYU Hospital for Joint Diseases, 301 E 17th St. \#1402, New York, NY, 10003, United States.

${ }^{\mathrm{b}}$ Orthopaedic Surgery, University of Calgary, 3330 Hospital Drive NW, Calgary, AB, T2N 4N1, Canada.

${ }^{c}$ Neurological Surgery, Northwestern University - Feinberg School of Medicine, 303 E Chicago Ave., Chicago, IL, 60611, United States.

${ }^{\mathrm{d}}$ Neurosurgery, University of Virginia Medical Center, 1215 Lee St., Charlottesville, VA, 22903, United States.

${ }^{\mathrm{e}}$ Orthopaedic Surgery, Hospital for Special Surgery, 535 E 70th St., New York, NY, 10021, United States.

${ }^{\mathrm{f}}$ Orthopaedic Surgery, University of California Davis, 4860 Y St., Sacramento, CA, 95817, United States.

${ }^{\mathrm{g}}$ San Diego Center for Spinal Disorders, 4130 La Jolla Village Dr., La Jolla, CA, 92037, United States.

${ }^{\mathrm{h}}$ Orthopaedic Surgery, Oregon Health and Science University, 3181 S.W. Sam Jackson Park Road, Portland OR, 97239

${ }^{i}$ Neurosurgery, University of California San Francisco, 505 Parnassus Ave., San Francisco, CA, 94143, United States.

${ }^{\mathrm{j}}$ ISSGF, 4 West Dry Creek Cir., Littleton, CO, 80120, United States.
}

\section{Corresponding Author:}

Peter G. Passias, M.D.

New York Spine Institute

361 Merrick Avenue

Westbury, New York 11590

Phone: 516-357-8777

Fax: 516-357-0087

Email: Peter.Passias@nyumc.org 


\section{Abstract:}

2 Background Context: Cervical Deformity (CD) is prevalent among patients with adult

3 spinal deformity (ASD). The effect of baseline cervical alignment on achieving optimal

4 thoracolumbar alignment in ASD surgery is unclear.

5

6 Purpose: This study assesses the relationship between pre-operative cervical spinal

7 parameters and global alignment following thoracolumbar ASD surgery at 2-year follow up.

9 Study Design/Setting: A retrospective review of a multi-center, prospective database.

11 Patient Sample: Surgical ASD patients with 2-year follow-up and cervical x-rays.

13 Outcome Measures: The outcome measure was radiographic parameters and self-reported 14 HRQL measures (SF-36, ODI and SRS-22).

16 Methods: Surgical ASD patients over the age of 18 with scoliosis $\geq 20^{\circ}$ and one of the

17 following radiographic parameters were included: $S V A \geq 5 \mathrm{~cm}$, pelvic tilt $\geq 25^{\circ}$ or thoracic

18 kyphosis $>60^{\circ}$. SRS-Schwab sagittal modifiers (PT, GA, PI-LL) were assessed at 2-year post-

19 op as either normal ("0") or abnormal ("+" or "++"). Patients were classified in the Aligned

20 Group (AG) or Malaligned Group (MG) at 2-year follow-up if all 3 sagittal modifiers were

21 normal or abnormal, respectively. Patients were assessed for CD based on the following

22 criteria: C2-C7 SVA $>4 \mathrm{~cm}, \mathrm{C} 2-\mathrm{C} 7 \mathrm{SVA}<4 \mathrm{~cm}$, cervical kyphosis ( $\mathrm{CL}>0$ ), cervical lordosis (CL

$23<0$ ), any deformity (C2-C7 SVA $>4 \mathrm{~cm}$ OR CL $>0$ ), and both CD (C2-C7 SVA >4cm AND CL >0). 
1 Univariate testing was performed using t-tests or chi square, looking at the following pre-

2 op parameters: CD, C2-C7 SVA, C2-T3 SVA, CL, T1S, T1S-CL, C2-T3 angle, LL, TK, PT, C7-S1

3 SVA, and PI-LL. No study funding sources are related to this clinical study. The

4 International Spine Study Group (ISSG) is funded through research grants from DePuy-

5 Synthes and individual donations.

6

7 Results: 104 patients met initial inclusion criteria with 70 in the AG group and 34 in MG.

8 Pre-op, patients in the MG group had a higher cervical lordosis (11.7 vs 4.9, p=0.03), higher

9 C2-T3 angle (13.59 vs $4.9 \mathrm{p}=0.01)$, higher PT $(\mathrm{p}<0.0001)$, higher SVA $(\mathrm{p}<0.0001)$, and

10 higher PI-LL $(\mathrm{p}<0.0001)$ compared to the AG group. Interestingly, the prevalence of CD at

11 baseline was similar for both groups. There was no statistically significant difference

12 among groups in the amount of improvement over 2 years on the ODI or the SF-36 PCS.

13

14 Conclusions: Patients with sagittal spinal mal-alignment associated with significant cervical

15 compensatory lordosis are at increased risk of realignment failure at 2 year follow up.

16 Assessment of the degree of cervical compensation may be helpful in preoperative

17 evaluation to assist in realignment outcome prediction.

18

19

20

21

22

23 
3 Adult spinal deformities most commonly occur in the thoracolumbar spine. As such,

4 previous investigations have focused on this region and placed less emphasis on alignment

5 changes that occur at the cervico-thoracic junction and above. More recently, however,

6 several series have shown a high prevalence of cervical deformity among patients being

7 treated primarily for adult thoracolumbar spinal deformity, which has been attributed to

8 the interconnected relationship between the various portions of the mobile spine and their

9 collective contribution to global sagittal alignment.[1, 2]

11 Previous authors have documented that the cervical spine has the capacity to compensate

12 for changes in thoracolumbar alignment because of its flexibility.[3-6] These observed

13 cervical alignment adjustments were found to be dependent on the pre-operative sagittal

14 alignment characteristics of the thoracolumbar spine, but following surgery with

15 instrumentation, this inverse correlation between cervical and thoracic kyphosis was not

16 appreciated presumably due to increased rigidity.[7] The presence of pre-operative

17 cervical deformity has also been shown to adversely affect clinical outcomes following

18 surgical correction of adult thoracolumbar spinal deformities at early follow-up.[7] No

19 study to date has clearly assessed the impact on clinical outcomes of cervical deformity

20 after thoracolumbar deformity surgery. The reason for this negative clinical impact

21 remains uncertain, as does its persistence with longer follow-up. Specifically, it is unclear

22 whether the baseline cervical alignment plays a role in achieving optimal thoracolumbar

23 alignment after surgical treatment, and what the associated clinical impact is at 2 years. 
1 As sagittal malalignment is a major source of pain and disability post-operatively, it is

2 important to investigate this problem with respect to the prevention of secondary cervical

3 spine disorders, particularly because the severity of symptoms has been shown to increase

4 in a linear fashion with progressive sagittal malalignment.[6] Therefore, if certain

5 radiographic parameters of the cervical spine were identified as risk factors for sagittal

6 malalignment after thoracolumbar surgery, surgeons could potentially accommodate for

7 this in surgical planning.

8

9 The present study attempts to assess the relationship between pre-operative cervical spinal parameters and post-operative global alignment. Characteristics of global alignment

11 were assessed using the SRS-Schwab Classification, which correlates radiographic

12 parameters with pain and disability in ASD [8] and has been shown to have the capability to

13 reliably predict patient disability and patient preference for operative versus nonoperative

14 treatment.[8] Therefore, this study used the SRS-Schwab Classification to sort patients into

15 a malaligned or aligned group and to determine risk factors for post-operative sagittal

16 spinal pelvic malalignment following thoracolumbar ASD surgery at 2-year follow-up.

17

Materials and Methods:

Study design and inclusion criteria:

21 This is a multi-center, retrospective review of prospectively acquired data for consecutive

22 patients who underwent surgical correction for sagittal ASD at eleven sites from 2008-

23 2011. All participating sites are a part of the International Spine Study Group (ISSG). Prior 
to study initiation, Institutional Review Board approval was obtained. Inclusion criteria were patients over 18 years of age with scoliosis $\geq 20^{\circ}$, SVA $\geq 5 \mathrm{~cm}$, pelvic tilt $\geq 25^{\circ}$, or thoracic kyphosis $>60^{\circ}$ who received surgical treatment for a documented spinal deformity. Included subjects also had complete demographic, radiographic, health-related quality of life HRQOL) surveys at baseline and 2-year follow-up as well as operative data. Exclusion criteria were patients with a lack of visibility of the cervical spine on their x-rays.

Full-length free-standing lateral spine radiographs (36" cassette) at baseline, 6 weeks, 3 months, 1 year, and 2 year follow-up were analyzed using validated software[9, 10] (Spineview®, ENSAM, Laboratory of Biomechanics, Paris, France). All radiographic measures were performed at a central location based on standard techniques[11] and included: coronal Cobb angles of thoracic and lumbar curves, thoracic kyphosis (TK, T4T12; Cobb angle between superior endplate of T4 and inferior endplate of T12), LL (Cobb angle between superior endplate of L1 and superior endplate of S1), SVA (C7 plumbline relative to S1), pelvic tilt (PT), and the mismatch between pelvic incidence and lumbar lordosis (PI-LL).

Additional cervical radiographic parameters included cervical lordosis (CL) for C2-C7, C2T3 angle, cervical SVA measured from C2-C7, T1 slope (T1S), and the mismatch between T1 slope and cervical lordosis (T1S-CL). Patients were assessed for cervical deformity (CD) at baseline and at 2-year follow-up based on the following criteria: C2-C7 SVA >4cm, C2-C7 SVA $<4 \mathrm{~cm}$, cervical kyphosis ( $\mathrm{CL}>0$ ), cervical lordosis ( $\mathrm{CL}<0$ ), any deformity ( $\mathrm{C} 2$-C7 SVA $>4 \mathrm{~cm}$ OR CL $>0$ ), and both CD (C2-C7 SVA $>4 \mathrm{~cm}$ AND CL $>0$ ). 
2 Based on the above radiographic parameters, patients were stratified by the SRS-Schwab

3 adult spinal deformity classification.[12] SRS-Schwab sagittal modifiers (PT, GA, PI-LL)

4 were assessed at 2-year post-op as either normal ("0") or abnormal ("+" or "++"). Patients

5 were assigned the Aligned Group (AG) or Malaligned Group (MG) if all 3 sagittal modifiers

6 were normal or abnormal, respectively. (Figure 1)

7

8 Data that were collected and utilized for this study included patient demographics, patient-

9 reported health-related quality of life scores such as ODI and SF-36 that were analyzed

10 before and after surgery. Data were uploaded into an electronic data capture system and

11 processed through the International Spine Study Group (ISSG) data management center.

12 The International Spine Study Group (ISSG) is funded through research grants from DePuy-

13 Synthes and individual donations.

14

15 Statistical analysis:

16

17 Statistical analysis was performed using Stata v13 (StataCorp, College Station, TX). The two 18 groups (malaligned vs. aligned based on SRS-Schwab sagittal parameters) were compared

19 in regards to patient demographics, surgical data, HRQOL, cervical and thoracic sagittal

20 radiographic parameters (C2-C7 SVA, C2-T3 SVA, CL, T1S, T1S-CL, C2-T3 angle) at baseline

21 and two years, and baseline lumbo-pelvic radiographic parameters (LL, TK, PT, C7-S1 SVA,

22 and PI-LL) using t-test and chi-square tests as appropriate. The relationship between 
cervical alignment and SRS-Schwab parameters was assessed using Pearson correlation coefficient. The level of significance was $\mathrm{p}<0.05$.

\section{Results:}

184 patients initially met inclusion criteria and had completed 2-year follow-up and cervical spine x-rays. Specifically, at the two-year follow-up, 70 patients had normal alignment on all SRS-Schwab sagittal modifier parameters (aligned group), and 34 patients had moderate or severe residual deformity on all Schwab SRS sagittal parameters (malaligned group). Of the original cohort, 80 patients were not included as they did not fit in either the malaligned or aligned groups, according to the sagittal modifier parameters.

(2)

Patient demographics and surgical data for the two groups show that on average, patients who failed to reach alignment on all three SRS-Schwab sagittal modifiers were older (58.9 years vs. 48.44 years, $\mathrm{p}=0.0017$ ), had a higher number of co-morbidities (Charlson Comorbidity Index 1.79 vs. 1.07, p=0.034), and a higher incidence of diabetes $(11.8 \%$ vs. $1.4 \%$ ) than their counterparts who were sagittally aligned at 2 years post-operatively. The two groups were similar in regards to smoking status and incidence of osteoporosis. On average, patients in the malaligned group had less levels fused (8.6 vs. 10.6, p=0.0131) and were less likely to have had an osteotomy performed $(32.3 \%$ vs. $70 \%, p=0.0001)$. There was no statistically significant difference between the two groups in terms of surgical approach (two-staged vs. posterior only) or the number of patients who underwent 3column osteotomies (Table 1). 
2 Table 2 shows the comparison of pre-operative sagittal alignment between the two groups.

3 Looking at cervical sagittal parameters, patients who were classified as "malaligned" based

4 on the 2-year SRS-Schwab sagittal modifiers had a higher pre-operative cervical lordosis

5 compared to the aligned group. This was true when looking at the C2-C7 lordosis $\left(11.66^{\circ}\right.$

6 vs. $\left.4.89^{\circ}, \mathrm{p}=0.03\right)$ and the C2-T3 lordosis $\left(13.59^{\circ}\right.$ vs. $\left.4.93^{\circ}, \mathrm{p}=0.015\right)$. There was no

7 statistically significant difference between the two groups when looking at the baseline C2-

8 C7 SVA, C2-T3 SVA, T1 slope and T1 slope-cervical lordosis (T1S-CL). The two groups were

9 similar in regards to their baseline thoracic kyphosis. Figures 2 and 3 display aligned and

10 malaligned patients according to cervical and thoracolumbar radiographic parameters.

12 When looking at pre-operative pelvic and global sagittal parameters, the malaligned group

13 had greater baseline deformity, as indicated by their higher pelvic tilt (33.43 vs. 15.81,

$14 \mathrm{p}<0.0001)$, lower lumbar lordosis (34.48 vs. 45.63, $\mathrm{p}=0.0315)$, greater mismatch between

15 pelvic incidence and lumbar lordosis (PI-LL, 27.03 vs. 1.68, $\mathrm{p}<0.0001$ ), and greater C7-S1

$16 \operatorname{SVA}(124.9$ vs. $36.7, \mathrm{p}<0.0001)$.

17

18 Cervical and thoracic sagittal alignment comparison between the two groups 2 years post-

19 operatively show that patients who did not reach alignment on the SRS-Schwab sagittal

20 modifiers had, on average, more post-operative lordosis between C2 and T3 (5.9 vs. -1.41,

$21 \mathrm{p}=0.0182$ ) and less thoracic kyphosis (39.59 vs. 46.46, $\mathrm{p}=0.0459$ ) when compared with

22 their counterparts who were aligned. There was a trend towards higher post-operative C2-

23 C7 SVA (39.61 vs. 32.24mm, p=0.051) and higher C2-T3 SVA (68.47 vs. 38.95mm, p=0.068) 
1 in the malaligned groups. The two groups were similar in regards to their post-operative

2 cervical lordosis and T1 slope.As shown in Table 4, the change in HRQOL outcomes

3 between baseline and 2-year post-op for the two groups was similar, with both groups

4 experiencing an improvement on ODI ( 11.09 vs. $12.97, \mathrm{p}=0.6)$, SRS ( 0.80 vs. $0.615, \mathrm{p}=0.2$ ),

5 and SF-36 physical (5.11 vs. $6.98, \mathrm{p}=0.45)$.

6

7 The Pearson's test shows a small, statistically significant positive correlation between

8 baseline cervical lordosis (as measured by both C2-C7 and C2-T3), post-operative pelvic

9 tilt, and C7 SVA (Table 5). Results also show a small positive correlation in two sets of radiographic parameters: between post-operative C2-T3 lordosis and post-op pelvic tilt/C7

11 SVA, and between post-operative C2-C7 lordosis and C7 SVA. There was no statistically

12 significant correlation between baseline or post-operative cervical lordosis and post-

13 operative PI-LL.

\section{Discussion}

17 Due to the complex anatomy of the cervical spine and the unequivocal interrelationship

18 between the cervical and thoracolumbar spine, it is important to investigate the

19 compensation that occurs after thoracolumbar surgery. Although the entire spine functions

20 as a single unit, depending on the pre-operative sagittal alignment, previous history of

21 cervical spine surgery involving fusion and/or instrumentation, and the degree of

22 thoracolumbar correction, patients compensate to different extents. Furthermore, regional 
1 changes in spinal alignment following surgery affect the alignment of the cervical region in

2 the early postoperative period.[13-15] Blondel et al reported a reciprocal reduction of

3 cervical lordosis following lumbar pedicle subtraction osteotomies.[14] In this series, we

4 comparatively evaluated two groups, those who obtained and those who did not obtain

5 ideal SRS-Schwab alignment criteria post-operatively, in order to look for the difference in

6 the incidence of cervical deformity, HRQOL outcome, and the correlation between cervical

7 and thoracolumbar deformities pre-operatively and post-operatively.

9 As adult spinal deformity (ASD) often involves various pathologies with a wide range of radiographic and clinical presentations, researchers found import in developing an ASD

11 classification system that is easy to use, categorized based on clinical impact, and highly

12 reliable.[7] The SRS-Schwab classification system was developed using studies correlating

13 health-related quality of life (HRQOL) and radiographic outcomes, and has been shown to

14 have the capability to reliably predict patient disability and patient preference for

15 operative versus non-operative treatment.[8] It places high import on the role of pelvic

16 alignment in the maintenance of upright posture, and hence the development of the

17 concept of spinopelvic alignment.[8] Furthermore, the system provides a unique and invaluable service to surgeons as a guideline for surgical planning with its global alignment

19 and sagittal modifiers (PI-LL, PT, and SVA). Therefore, this study utilized the SRS-Schwab sagittal modifiers to categorize patients who underwent thoracolumbar surgery into

21 aligned and malaligned groups at two years following corrective surgery. In contrast to

22 adult thoracolumbar deformity, there is a lack of a universally accepted definition of

23 cervical deformity. As such, for the purposes of our study we utilized a comprehensive 
1 group of radiographic parameters, with strict values for inclusion, until these parameters

2 are more clearly established in the literature. This was done for the purposes of being more

3 inclusive of all variations of cervical deformity while at the same time only including

4 patients with true cervical deformity.

5

6 This series demonstrated distinct differences between patients who obtained ideal

7 alignment relative to those who did not. In terms of demographics, patients in the

8 malaligned group at 2 years, as determined by the SRS-Schwab classification, were older

9 and had a higher comorbidity burden, representing a higher surgical risk population.

10 Surgically, the malaligned group had less levels fused than patients in the aligned group.

11 Additionally, the malaligned group was more likely to undergo osteotomies and presented

12 with greater overall thoracolumbar deformities. This is expected as osteotomies are often

13 reserved for patients with significant spinal deformities. However, it is interesting to note

14 that despite the performance of these osteotomies, their overall alignment remained

15 inferior at 2-year follow-up. The same group of patients also had higher overall baseline

16 thoracolumbar deformities, as evidenced by higher pelvic tilt, PI-LL mismatch, and SVA, all

$17 \mathrm{p}<0.0001$.

18

19 Not surprisingly, at 2-year post-op, the malaligned group continued to manifest statistically

20 different thoracolumbar deformities compared to the aligned group. However, their

21 baseline degree of cervical lordotic compensation improved to the point where the

22 difference in cervical lordosis between aligned and malaligned group was not statistically

23 significant. By our definition and categorization, the malaligned group was malaligned both 
1 prior to and after their thoracolumbar surgery. However, our analysis showed a more

2 significant improvement in cervical lordosis measurement in the malaligned group than the

3 aligned group, most likely a result of this cohort starting with larger baseline sagittal

4 deformity. Despite the difference in pre-operative and post-operative alignment, the

5 HRQOL score differences at 2-year post-op between the two groups were not statistically

6 significant. The HRQOL score difference was measured using ODI, SRS-22 and SF-36, which

7 are more specific to the lumbar spine rather than the cervical spine. Therefore, an analysis

8 of the HRQOL score based on the cervical spine such, as the Neck Disability Index (NDI),

9 could provide a more representative result on the relative effect of lumbar surgery on cervical spine health, as perceived by the patient. This is particularly relevant in light of our

11 findings of increased baseline cervical and cervico-thoracic lordosis.

13 Perhaps the most significant finding was that patients who were classified as "malaligned"

14 based on the 2-year SRS-Schwab sagittal modifiers had a higher pre-operative cervical and

15 cervico-thoracic lordosis compared to the aligned group, as defined by C2-C7 and C2-T3

16 lordosis measurements. Post-operatively, the malaligned cohort had persistence of

17 relatively increased C2-T3 lordosis, with a trend towards increased C2-C7 and C2-T3 SVA.

18 These significant findings persisted as being independent predictors of not reaching

19 optimal thoracolumbar alignment even after controlling for confounders. Overall these

20 findings are novel and can be interpreted as correlative evidence of a relationship between

21 cervical lordosis and greater thoracolumbar malalignment at 2 years post-operatively. In

22 order to further define the relationship between cervical compensatory lordosis and post-

23 operative thoracolumbar malalignment, the present study also analyzed for correlation 
1 between all patients - both aligned and malaligned groups-and radiographic parameters,

2 and found a small positive correlation between baseline cervical lordosis and post-

3 operative PT and SVA, between post-operative C2-T3 lordosis and post-operative PT/SVA,

4 and between post-operative C2-C7 lordosis and post-operative SVA. Our results are

5 consistent with previous studies that have discussed a "chain of correlation" between

6 various regional alignments of the spinal column, including, cervical lordosis and thoracic

7 kyphosis, thoracic kyphosis and lumbar lordosis, lumbar lordosis and pelvic incidence, and

8 pelvic tilt and cervical lordosis.[16] However, this is the first series to document a

9 significant correlation between certain baselines as well as persistent two year increased

10 cervico-thoracic lordosis, and the presence of sub-optimal thoracolumbar/pelvic alignment

11 at two years following corrective thoracolumbar ADS surgery. Further studies with larger

12 sample sizes are necessary in order to determine if cervical lordotic compensation is a

13 predictor of two-year malalignment. In addition, potentially quantifying degree of cervical

14 lordotic compensation as hyperlordosis may be useful in similar future studies, yet

15 requires further investigation as there are no universally established parameters for the

16 definition of the latter.

17

18 Although our data and analysis show valuable information, our study is limited in its

19 retrospective design, and therefore a prospective study is warranted. Although

20 representing a large cohort relative to the majority of ADS series, our patient sample

21 remains less than ideal for analyzing variables other than our primary outcomes. Lastly,

22 spinal alignment is not independent of the entire body alignment, including horizontal gaze 
and lower extremity posturing, and future studies are required to account for changes in these other regions.

\section{Conclusion}

The interdependence between the cervical spine and lumbar spine is not only compelling, but also unequivocal. Therefore, for patients undergoing either cervical or lumbar spine surgery, it is imperative that their healthcare providers are aware of the surgery's impact on the region of the spine not undergoing surgery. This study identified preoperative cervical degree of lordotic compensation and higher C2-T3 angle as risk factors for sagittal malalignment after thoracolumbar surgery. Further, the presence of sub-optimal thoracolumbar and pelvic alignment at two years following corrective thoracolumbar ADS surgery was identified. Patients who remained malaligned at 2-year post-op (malaligned group) also had worse malalignment with higher PT, PI-LL, and SVA despite undergoing more extensive and risky osteotomies. With this information, surgeons could utilize radiographic parameters of the cervical spine, specifically C2-C7 lordosis, the C2-T3 lordosis, and C2-T3 angle, to identify patients who are at risk for sagittal malalignment after thoracolumbar surgery, to educate patients, and to plan for surgery accordingly, potentially obviating the consideration for osteotomies. Unfortunately, this study is limited due to its retrospective design, and therefore, prospective, randomized trials are needed to verify the results of this study. 


\section{References}

1. Ha Y, Schwab F, Lafage V, et al. Reciprocal changes in cervical spine alignment after corrective thoracolumbar deformity surgery. European spine journal : official publication of the European Spine Society, the European Spinal Deformity Society, and the European Section of the Cervical Spine Research Society. 2014;23(3):552-9.

2. Ilharreborde B, Vidal C, Skalli W, Mazda K. Sagittal alignment of the cervical spine in adolescent idiopathic scoliosis treated by posteromedial translation. European spine journal : official publication of the European Spine Society, the European Spinal Deformity Society, and the European Section of the Cervical Spine Research Society. 2013;22(2):330-7.

3. Lafage V, Smith JS, Bess S, et al. Sagittal spino-pelvic alignment failures following three column thoracic osteotomy for adult spinal deformity. European Spine Journal. 2012;21(4):698704.

4. Klineberg E, Schwab F, Ames C, et al. Acute reciprocal changes distant from the site of spinal osteotomies affect global postoperative alignment. Advances in orthopedics. 2011;2011.

5. Lafage V, Schwab F, Vira S, Patel A, Ungar B, Farcy J-P. Spino-pelvic parameters after surgery can be predicted: a preliminary formula and validation of standing alignment. Spine. 2011;36(13):1037-45.

6. Glassman SD, Bridwell K, Dimar JR, Horton W, Berven S, Schwab F. The impact of positive sagittal balance in adult spinal deformity. Spine. 2005;30(18):2024-9.

7. Ha Y, Schwab F, Lafage V, et al. Reciprocal changes in cervical spine alignment after corrective thoracolumbar deformity surgery. European Spine Journal. 2013:1-8.

8. Bess S. Classifications for Adult Spinal Deformity and Use of the Scoliosis Research Society-Schwab Adult Spinal Deformity Classification. Neurosurg Clin N Am. 2013;24(2):18593.

9. Champain S, Benchikh K, Nogier A, Mazel C, Guise JD, Skalli W. Validation of new clinical quantitative analysis software applicable in spine orthopaedic studies. European spine journal : official publication of the European Spine Society, the European Spinal Deformity Society, and the European Section of the Cervical Spine Research Society. 2006;15(6):982-91.

10. Rillardon L, Levassor N, Guigui P, et al. [Validation of a tool to measure pelvic and spinal parameters of sagittal balance]. Revue de chirurgie orthopedique et reparatrice de l'appareil moteur. 2003;89(3):218-27.

11. O'Brien MF, Kuklo TR, Blanke K, Lenke L, eds. Spinal Deformity Study Group Radiographic Measurement Manual. Memphis, TN: Medtronic Sofamor Danek; 2005.

12. Schwab F, Ungar B, Blondel B, et al. Scoliosis Research Society-Schwab adult spinal deformity classification: a validation study. Spine. 2012;37(12):1077-82.

13. Smith JS, Shaffrey CI, Lafage V, et al. Spontaneous improvement of cervical alignment after correction of global sagittal balance following pedicle subtraction osteotomy: Clinical article. Journal of Neurosurgery: Spine. 2012;17(4):300-7.

14. Blondel B, Schwab F, Bess S, et al. Posterior global malalignment after osteotomy for sagittal plane deformity: it happens and here is why. Spine. 2013;38(7):E394-E401.

15. Passias PG, Wang S, Zhao D, Wang S, Kozanek M, Wang C. The Reversibility of Swan Neck Deformity in Chronic Atlantoaxial Dislocations. Spine. 2013;38(7):E379-E85.

16. Scheer JK, Tang JA, Smith JS, et al. Cervical spine alignment, sagittal deformity, and clinical implications: a review. Journal of Neurosurgery: Spine. 2013;19(2):141-59. 


\section{Figure Legends:}

2 Figure 1. SRS-Schwab classification of adult spinal deformity

3 Figure 2: Preoperative and post—operative changes in radiographic parameters, including the

4 cervical spine, following surgical treatment.

5 Figure 3: Clinical preoperative and post—operative changes in radiographic parameters for 6 thoracolumbar and cervical deformities. 
Table 1. Summary of patient population

\begin{tabular}{|l|l|l|l|}
\hline & $\begin{array}{l}\text { 2yr Aligned } \\
\text { Group (n=70) }\end{array}$ & $\begin{array}{l}\text { 2yr Malaligned } \\
\text { Group (n=34) }\end{array}$ & p-value \\
\hline Age & 48.44 & 58.90 & 0.0017 \\
\hline CCI & 1.07 & 1.79 & 0.034 \\
\hline Diabetes & $1.4 \%$ & $11.8 \%$ & 0.021 \\
\hline Osteoporosis & $8.6 \%$ & $14.7 \%$ & 0.34 \\
\hline Smoker & $15.9 \%$ & $9.4 \%$ & 0.384 \\
\hline Levels fused & 10.6 & 8.6 & 0.0131 \\
\hline Interbody & $45.7 \%$ & $64.7 \%$ & 0.07 \\
\hline Osteotomy (all) & $32.3 \%$ & $70 \%$ & 0.0001 \\
\hline Osteotomy (3-column) & $13 \%$ & $17.6 \%$ & 0.533 \\
\hline Approach (two-stage & $42.8 \%$ & $52.94 \%$ & 0.333 \\
\hline vs. posterior only) & & & \\
\hline
\end{tabular}


Table 2. Comparison of pre-operative sagittal alignment between the two groups

\begin{tabular}{|l|l|l|l|}
\hline $\begin{array}{l}\text { Pre-op Sagittal } \\
\text { Parameters }\end{array}$ & 2yr Aligned & 2yr Malaligned & p-value \\
\hline C2-C7 SVA & $32.1 \pm 1.8$ & $35.4 \pm 3.2$ & 0.34 \\
\hline C2-T3 SVA & $54.3 \pm 2.7$ & $61.1 \pm 4.6$ & 0.17 \\
\hline CL & $4.89 \pm 1.7$ & $11.66 \pm 2.7$ & 0.03 \\
\hline T1S & $22.3 \pm 1.46$ & $25.8 \pm 2.17$ & 0.18 \\
\hline T1S-CL & $17.36 \pm 1.06$ & $14.32 \pm 2.1$ & 0.15 \\
\hline C2-T3 angle & $4.93 \pm 1.94$ & $13.59 \pm 3.0$ & 0.015 \\
\hline LL & $45.63 \pm 2.5$ & $35.48 \pm 4.3$ & 0.0315 \\
\hline TK & $34.95 \pm 2.28$ & $30.3 \pm 3.55$ & 0.26 \\
\hline PT & $15.81 \pm 1.08$ & $33.43 \pm 1.6$ & $<0.0001$ \\
\hline C7-S1 SVA & $36.7 \pm 7.2$ & $124.9 \pm 12.5$ & $<0.0001$ \\
\hline PI-LL & $1.68 \pm 2.18$ & $27.03 \pm 3.88$ & $<0.0001$ \\
\hline
\end{tabular}


Table 3. Sagittal parameters for the two groups

\begin{tabular}{|l|l|l|l|}
\hline $\begin{array}{l}\text { 2yr Sagittal } \\
\text { Parameters }\end{array}$ & $\begin{array}{l}\text { 2yr Aligned } \\
\text { Group (n=70) }\end{array}$ & $\begin{array}{l}\text { 2yr Malaligned } \\
\text { Group (n=34) }\end{array}$ & p-value \\
\hline C2-C7 SVA & $32.24 \pm 1.72$ & $39.61 \pm 3.9$ & 0.051 \\
\hline C2-T3 SVA & $58.95 \pm 2.6$ & $68.57 \pm 5.1$ & 0.068 \\
\hline CL & $4.3 \pm 1.92$ & $8.8 \pm 3.01$ & 0.196 \\
\hline T1S & $26.7 \pm 1.49$ & $31.5 \pm 2.44$ & 0.0821 \\
\hline T1S-CL & $22.44 \pm 1.2$ & $22.73 \pm 2.32$ & 0.9 \\
\hline C2-T3 angle & $-2.41 \pm 1.9$ & $5.9 \pm 3.1$ & 0.0182 \\
\hline TK (T2-T12) & $46.46 \pm 1.9$ & $39.59 \pm 2.85$ & 0.0459 \\
\hline
\end{tabular}


Table 4. Changes in HRQOL outcomes between baseline and 2-year post-op for patient population

\begin{tabular}{|c|c|c|c|}
\hline $\begin{array}{c}\text { HRQOL Difference } \\
\text { Baseline to 2yr }\end{array}$ & $\begin{array}{c}\text { 2yr Aligned } \\
\text { Group (n=70) }\end{array}$ & $\begin{array}{c}\text { 2yr Malaligned } \\
\text { Group (n=34) }\end{array}$ & p-value \\
\hline ODI & -11.09 & -12.97 & 0.60 \\
\hline SRS & 0.80 & 0.615 & 0.2 \\
\hline SF-36 physical & 6.98 & 5.11 & 0.45 \\
\hline
\end{tabular}


Table 5. Relationship between baseline and post-op spinal parameters

\begin{tabular}{|c|c|c|c|c|c|c|}
\hline & $\begin{array}{c}2 \mathrm{yr} P \mathrm{PT} \\
\quad \mathrm{r}\end{array}$ & $\begin{array}{l}2 \text { yr PT } \\
\text { p-value }\end{array}$ & 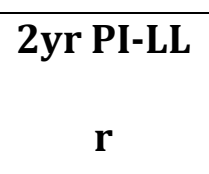 & $\begin{array}{l}\text { 2yr PI-LL } \\
\text { p-value }\end{array}$ & $\begin{array}{c}\text { 2yr C7 SVA } \\
\text { r }\end{array}$ & $\begin{array}{c}\text { 2yr C7 SVA } \\
\text { p-value }\end{array}$ \\
\hline \multicolumn{7}{|c|}{ Baseline lordosis } \\
\hline $\mathrm{C} 2-\mathrm{C} 7$ & 0.2419 & 0.0134 & 0.1074 & 0.2779 & 0.2517 & 0.0099 \\
\hline C2-T3 & 0.2599 & 0.0077 & 0.1076 & 0.2770 & 0.3196 & 0.0009 \\
\hline \multicolumn{7}{|c|}{ 2-year lordosis } \\
\hline C2-C7 & 0.1335 & 0.1766 & -0.0010 & 0.99 & 0.2385 & 0.0148 \\
\hline C2-T3 & 0.2274 & 0.0203 & 0.0663 & 0.5040 & 0.3314 & 0.0006 \\
\hline
\end{tabular}


Magnitude of Cervical Lordotic Compensation and C2-T3 Angle are Correlated to Increased Risk of Postoperative Sagittal Spinal Pelvic Malalignment in Adult Thoracolumbar Deformity Patients at 2-year Follow-up

Figure 1. SRS-Schwab classification of adult spinal deformity 


\section{Coronal Curve Types Sagittal Modifiers}

T: Thoracic only

with lumbar curve $<30^{\circ}$

L: TL / Lumbar only with thoracic curve $<30^{\circ}$

D: Double Curve

with $\mathrm{T}$ and $\mathrm{TL} / \mathrm{L}$ curves $>30^{\circ}$

N: No Major Coronal Deformity

all coronal curves $<30^{\circ}$

\section{PI minus LL}

0 : within $10^{\circ}$

+ : moderate $10-20^{\circ}$

++ : marked $>20^{\circ}$

Global Alignment

$0:$ SVA $<4 \mathrm{~cm}$

$+:$ SVA 4 to $9.5 \mathrm{~cm}$

$++:$ SVA $>9.5 \mathrm{~cm}$

Pelvic Tilt

$0:$ PT $<20^{\circ}$

+ : PT 20-30

++ : $\mathrm{PT}>30^{\circ}$ 


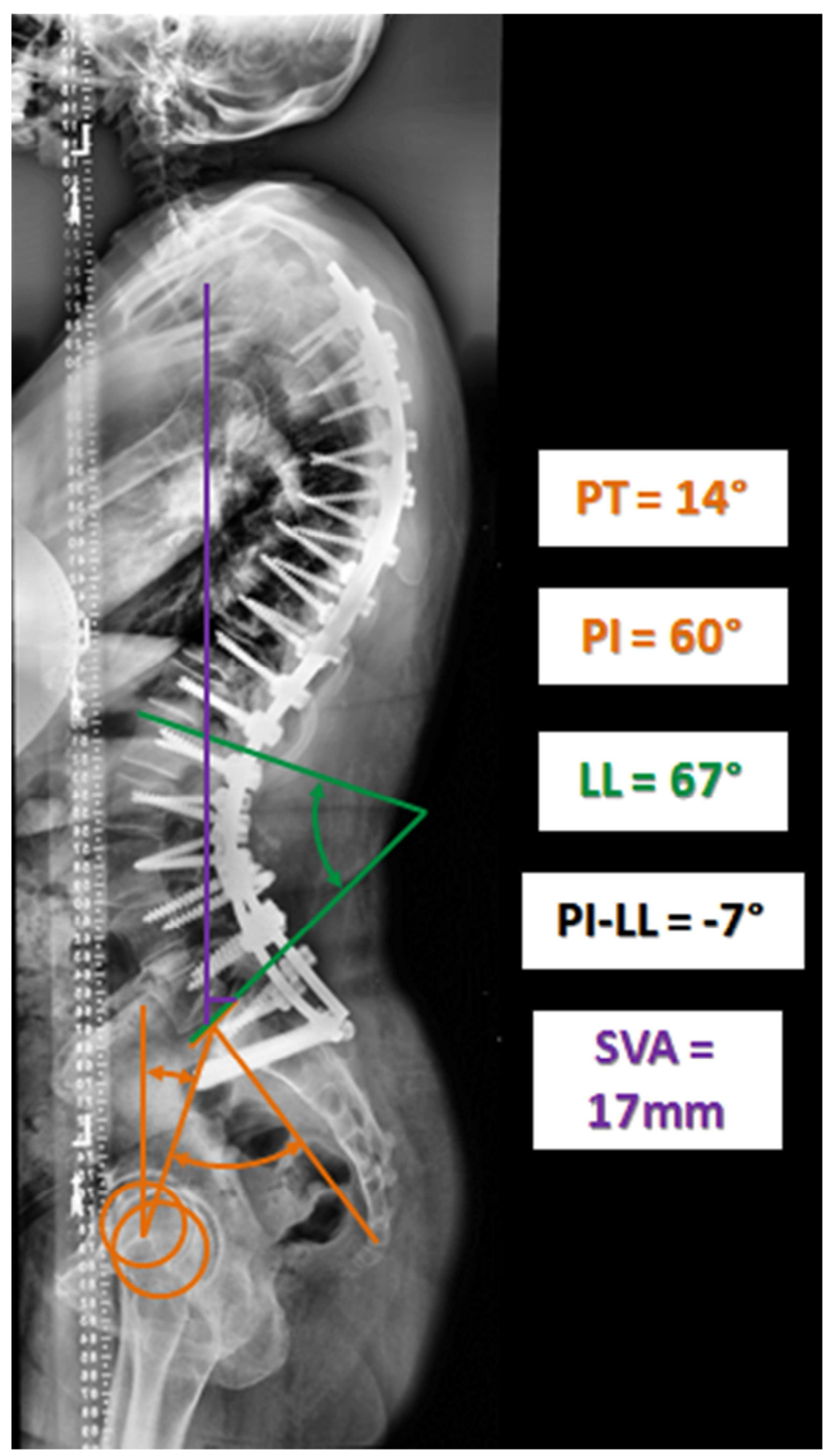




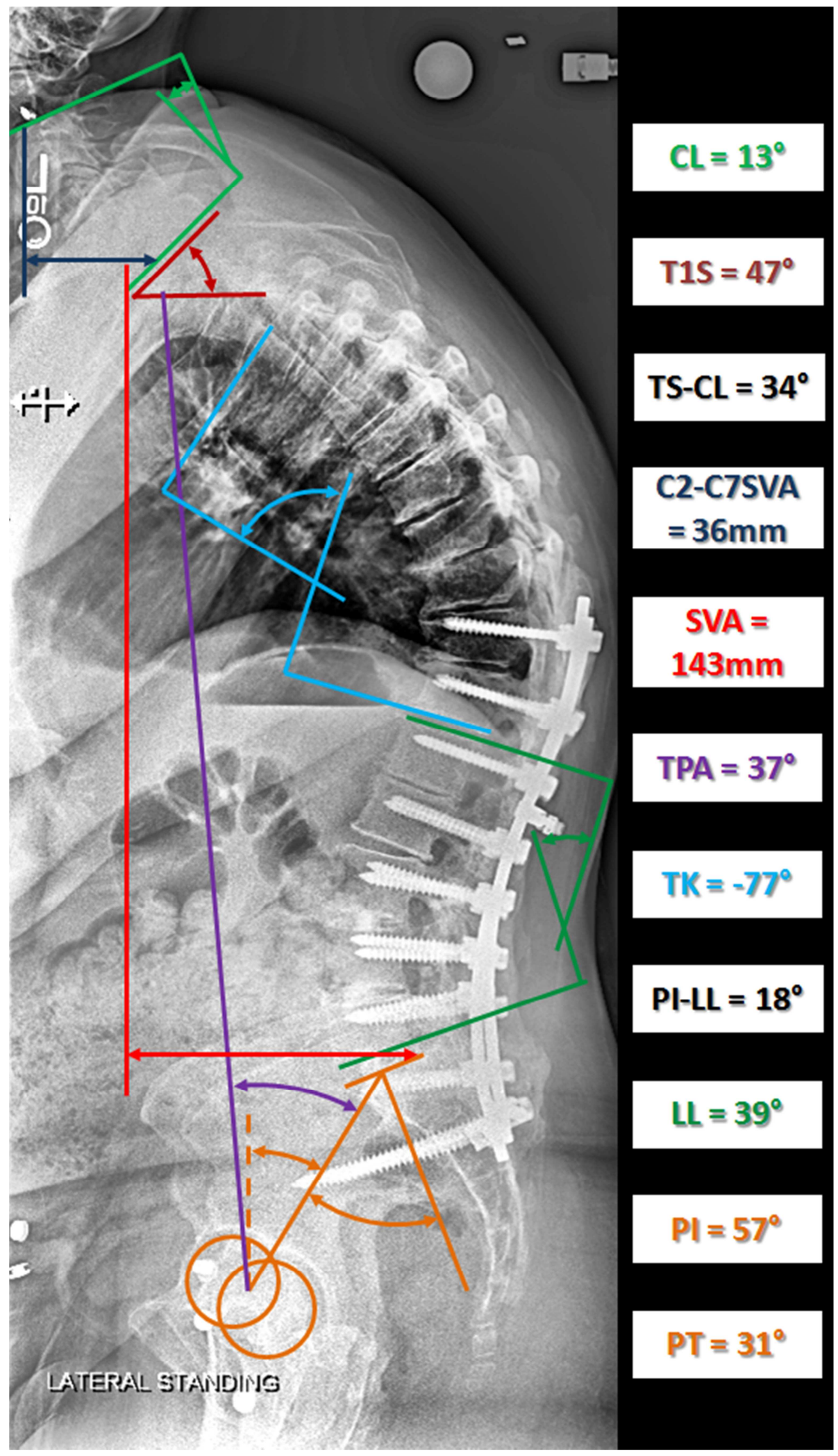

